\title{
STABILITY OF EQUILIBRIA OF NEMATIC LIQUID CRYSTALLINE POLYMERS*
}

\author{
Dedicated to Professor Peter D. Lax on the occasion of his 85 th birthday \\ Hong Zhou \\ Department of Applied Mathematics \\ Naval Postgraduate School, Monterey, CA 93943, USA \\ E-mail: hzhou@nps.edu \\ Hongyun Wang \\ Department of Applied Mathematics and Statistics \\ University of California, Santa Cruz, CA 95064, USA \\ E-mail: hongwang@soe.ucsc.edu
}

\begin{abstract}
We provide an analytical study on the stability of equilibria of rigid rodlike nematic liquid crystalline polymers (LCPs) governed by the Smoluchowski equation with the Maier-Saupe intermolecular potential. We simplify the expression of the free energy of an orientational distribution function of rodlike LCP molecules by properly selecting a coordinate system and then investigate its stability with respect to perturbations of orientational probability density. By computing the Hessian matrix explicitly, we are able to prove the hysteresis phenomenon of nematic LCPs: when the normalized polymer concentration $b$ is below a critical value $b^{*}(6.7314863965)$, the only equilibrium state is isotropic and it is stable; when $b^{*}<b<15 / 2$, two anisotropic (prolate) equilibrium states occur together with a stable isotropic equilibrium state. Here the more aligned prolate state is stable whereas the less aligned prolate state is unstable. When $b>15 / 2$, there are three equilibrium states: a stable prolate state, an unstable isotropic state and an unstable oblate state.
\end{abstract}

Key words equilibria; stability; nematic liquid crystalline polymers; hysteresis phenomenon

2000 MR Subject Classification $35 \mathrm{Kxx} ; 70 \mathrm{Kxx}$

\section{Introduction}

Since its discovery by Austrian botanical physiologist Friedrich Reinitzer in 1888, liquid crystals have spurted intensive experimental, theoretical and numerical studies $[1,5,6,18,31$, 39]. One notable example is that in 1991 Pierre-Gilles de Gennes was awarded the Nobel Prize

${ }^{*}$ Received September 30, 2011. This work is partially supported by the National Science Foundation and by the Office of Naval Research. 
in Physics "for discovering that methods developed for studying order phenomena in simple systems can be generalized to more complex forms of matter, in particular to liquid crystals and polymers".

Liquid crystals refer to a state of matter that has properties between those of a solid crystal and those of an isotropic liquid. For example, a liquid crystal may flow like an ordinary liquid, but its molecules may be oriented in some directions. Examples of liquid crystals can be found both in nature (e.g. solutions of soap and detergents) and in technological applications (e.g. electronic displays). There are many different types of liquid crystal phases, among which one of the most common phases is the nematic. In a nematic phase, the rod-shaped molecules have no positional order but they display long-range directional order with their long axes roughly aligned parallel to a common axis (which is called a "director"). Most nematics are uniaxial, meaning that they have one axis that is longer and preferred while the other two axes are equivalent. However, some nematics are biaxial which implies that the molecules also orient along a secondary axis in addition to orienting their long axis. Nematics can be easily aligned by applying an external magnetic or electric field. The optical properties of aligned nematics make them extremely useful in liquid crystal displays.

Liquid crystallinity in polymers may occur either by heating a polymer above its melting point (thermotropic LCPs) or by dissolving a polymer in a solvent (lyotropic LCPs). Liquid crystalline polymers combine the properties of liquid crystals with the properites of polymers. As a result, it is easy to guide LCPs by applying small external forces such as flows and it is easy to control their chemical and material parameters. LCPs exhibit certain anisotropy of the electrical, mechanical and magnetic properties with high flexibility or elasticity. They are also exceptionally unreactive and inert, and highly resistant to fire. Due to their various properties, LCPs have found more and more applications, including electrical and mechanical parts, food containers, and any other applications requiring chemical inertness and super strength. A main example of LCPs in solid form is high strength fibers which have been made into bullet-proof vests and airbags.

The theoretical studies of liquid crystals traced back more than 60 years ago. In 1949 Onsager [29] developed a statistical theory showing that simple exclude-volume repulsions between long rods are sufficient to create a liquid crystal. Onsager's theory is valid for dilute liquid crystals. Flory $[9,10]$ presented a lattice model and his theory yields good approximation for dense and highly ordered liquid crystals. Both the Onsager and Flory theories predict an isotropic-nematic phase transition when the concentration is high enough. In three publications Maier and Saupe [26-28] proposed a mean-field theory to explain the occurrence of a liquid crystal phase in low molecular weight materials based on the assumption that only induced dipolar forces are relevant for orientational ordering. An impressive signature for the popularity of the Maier-Saupe theory is its high number of citations (almost 4000 up to now) over the years [30]. In 1965 Landau and Ginzburg [22] developed a phenomenological theory for liquid crystals. Even though it has success in most cases, Landau-Ginzburg theory is not exact and it suffers failure in some cases [18]. One of the earliest constitutive theories of liquid crystals is the Leslie-Ericksen vector theory [7, 23] in 1970s. The Leslie-Ericksen theory is attractive for studying low molar-mass liquid crystals.

After Maier and Saupe and Flory's work, many new theories have been developed for liquid 
crystalline polymers. One of the most famous kinetic models for polymer liquid crystals was developed by Doi and Edwards [6] in 1980s and Hess [19]. The crucial idea of the Doi theory is to model the polymeric molecules as a suspension of rigid rodlike nematogens and then describe the ensemble with an orientational probability distribution function. The orientational distribution function is governed by a nonlinear Smoluchowski (or Fokker-Planck) equation which takes into account the hydrodynamic, Brownian and intermolecular forces.

In recent years, the Doi theory has attracted enormous mathematical attentions (for example, $[2-4,8,11-17,21,24,25,32-38])$. One mathematical advance in the understanding of the equilibrium states of the Doi model is the rigorous proof on that the equilibria of the Smoluchowski equation with the Maier-Saupe potential are uniaxial $[8,24,35]$. However, it seems more challenging to obtain a solid proof on the stability of the equilibria. The purpose of this paper is to circumvent this difficulty and seek a mathematical proof on the stability of the equilibria. The main result proven here is: all stable equilibria are either isotropic or highly aligned prolate uniaxial.

\section{Background and Formulation}

For reader's convenience, we start with the briefest glimpse of the Doi kinetic theory.

Let us denote the orientational direction of each rodlike molecule by a unit vector $\mathbf{m}$, the probability density function by $\rho(\mathbf{m})$. The evolution of the probability density function is described by the Smoluchowski equation [6]:

$$
\frac{\partial \rho}{\partial t}=D \frac{\partial}{\partial \mathbf{m}} \cdot\left(\frac{1}{k_{B} T} \frac{\partial U}{\partial \mathbf{m}} \rho+\frac{\partial \rho}{\partial \mathbf{m}}\right)
$$

where $\partial / \partial \mathbf{m}$ is the orientational gradient operator $[1], D$ the rotational diffusivity, $k_{B}$ the Boltzmann constant, and $T$ the absolute temperature. For simplicity, we set $k_{B} T=1$ which is equivalent to normalizing $U$ by $k_{B} T$.

For nematic polymers, the total potential consists of only the Maier-Saupe interaction potential

$$
U(\mathbf{m})=-b\langle\mathbf{m m}\rangle: \mathbf{m m},
$$

where the tensor product $\mathbf{m m}$ and tensor double contraction $\mathbf{A}: \mathbf{B}$ are defined as

$$
\mathbf{m m} \equiv\left[\begin{array}{lll}
m_{1} m_{1} & m_{1} m_{2} & m_{1} m_{3} \\
m_{2} m_{1} & m_{2} m_{2} & m_{2} m_{3} \\
m_{3} m_{1} & m_{3} m_{2} & m_{3} m_{3}
\end{array}\right]
$$

$$
\mathbf{A}: \mathbf{B} \equiv \operatorname{trace}(\mathbf{A B})
$$

In (2) the constant $b$ is proportional to the normalized polymer concentration and it describes the strength of inter-molecular interactions, and $\langle\mathbf{m m}\rangle$ denotes the second moment of the orientation distribution:

$$
\langle\mathbf{m m}\rangle \equiv \int_{\|\mathbf{m}\|=1} \mathbf{m m} \rho(\mathbf{m}) \mathrm{d} \mathbf{m},
$$

where $\rho(\mathbf{m})$ is the orientational probability density function of the ensemble of polymer rods, i.e., the probability density that a polymer rod has direction $\mathbf{m}$. 


\section{The Maier-Saupe potential in a properly selected coordinate system:}

Let us select a coordinate system such that the second moment $\langle\mathbf{m m}\rangle$ is diagonal:

$$
\langle\mathbf{m m}\rangle=\left[\begin{array}{ccc}
\left\langle m_{1}^{2}\right\rangle & 0 & 0 \\
0 & \left\langle m_{2}^{2}\right\rangle & 0 \\
0 & 0 & \left\langle m_{3}^{2}\right\rangle
\end{array}\right]=\left[\begin{array}{ccc}
s_{1}+\frac{1}{3} & 0 & 0 \\
0 & s_{2}+\frac{1}{3} & 0 \\
0 & 0 & s_{3}+\frac{1}{3}
\end{array}\right],
$$

where $s_{j} \equiv\left\langle m_{j}^{2}\right\rangle-\frac{1}{3}$ satisfies

$$
s_{1}+s_{2}+s_{3}=\left\langle m_{1}^{2}+m_{2}^{2}+m_{3}^{2}\right\rangle-1=0
$$

In this coordinate system, the Maier-Saupe potential takes the following expression:

$$
\begin{aligned}
U(\mathbf{m}) & =-b\left(\left\langle m_{1}^{2}\right\rangle m_{1}^{2}+\left\langle m_{2}^{2}\right\rangle m_{2}^{2}+\left\langle m_{3}^{2}\right\rangle m_{3}^{2}\right) \\
& =-\frac{b}{3}-b\left(s_{1} m_{1}^{2}+s_{2} m_{2}^{2}+s_{3} m_{3}^{2}\right) .
\end{aligned}
$$

The constant $(-b / 3)$ does not affect the dynamics or the stability of the nematic polymer ensemble. For simplicity, we drop the constant $(-b / 3)$. From $s_{1}+s_{2}+s_{3}=0$ and $m_{1}^{2}+m_{2}^{2}+m_{3}^{2}=$ 1 , we have

$$
\begin{aligned}
& s_{1}=\frac{-s_{3}}{2}-\frac{s_{2}-s_{1}}{2}, \\
& s_{2}=\frac{-s_{3}}{2}+\frac{s_{2}-s_{1}}{2}, \\
& m_{1}^{2}+m_{2}^{2}=1-m_{3}^{2} .
\end{aligned}
$$

Using these equations, we can rewrite the Maier-Saupe potential (3) as

$$
\begin{aligned}
U(\mathbf{m}) & =-b\left(\left(\frac{-s_{3}}{2}-\frac{s_{2}-s_{1}}{2}\right) m_{1}^{2}+\left(\frac{-s_{3}}{2}+\frac{s_{2}-s_{1}}{2}\right) m_{2}^{2}+s_{3} m_{3}^{2}\right) \\
& =-b \frac{3 s_{3}}{2}\left(m_{3}^{2}-\frac{1}{3}\right)-b \frac{\left(s_{2}-s_{1}\right)}{2}\left(m_{2}^{2}-m_{1}^{2}\right) \\
& =-\eta_{1}\left(m_{3}^{2}-\frac{1}{3}\right)-\eta_{2}\left(m_{2}^{2}-m_{1}^{2}\right),
\end{aligned}
$$

where

$$
\begin{aligned}
& \eta_{1} \equiv b \frac{3 s_{3}}{2}=b \frac{3}{2}\left(\left\langle m_{3}^{2}\right\rangle-\frac{1}{3}\right), \\
& \eta_{2} \equiv b \frac{\left(s_{2}-s_{1}\right)}{2}=b \frac{1}{2}\left\langle m_{2}^{2}-m_{1}^{2}\right\rangle .
\end{aligned}
$$

Note that for any orientational distribution $\rho(\mathbf{m})$, by selecting a proper coordinate system, we can always express the Maier-Saupe potential $U(\mathbf{m})$ in the form of (4) with $\eta_{1}$ and $\eta_{2}$ given by $(5)$ and $(6)$. In particular, $\rho(\mathbf{m})$ does not have to be an equilibrium distribution. 
In the absence of external field, the equilibrium distributions of a nematic polymer ensemble are described by the Boltzmann distribution [6]:

$$
\begin{aligned}
\rho^{(B Z)}\left(\mathbf{m} ; \eta_{1}, \eta_{2}\right) & =\frac{\exp [-U(\mathbf{m})]}{\int_{S} \exp [-U(\mathbf{m})] \mathrm{d} \mathbf{m}} \\
& =\frac{\exp \left[\eta_{1} m_{3}^{2}+\eta_{2}\left(m_{2}^{2}-m_{1}^{2}\right)\right]}{\int_{S} \exp \left[\eta_{1} m_{3}^{2}+\eta_{2}\left(m_{2}^{2}-m_{1}^{2}\right)\right] \mathrm{d} \mathbf{m}},
\end{aligned}
$$

where $S$ represents the unit sphere. It is clear that an equilibrium distribution is completely specified by $\left(\eta_{1}, \eta_{2}\right)$. To find an equilibrium distribution, we need to determine $\left(\eta_{1}, \eta_{2}\right)$. The governing equation for $\left(\eta_{1}, \eta_{2}\right)$ is obtained by combining the definition of $\left(\eta_{1}, \eta_{2}\right)$ and the Boltzmann distribution (7):

$$
\begin{aligned}
& \eta_{1}=b \frac{3}{2} \int_{S}\left(m_{3}^{2}-\frac{1}{3}\right) \rho^{(B Z)}\left(\mathbf{m} ; \eta_{1}, \eta_{2}\right) \mathrm{d} \mathbf{m}, \\
& \eta_{2}=b \frac{1}{2} \int_{S}\left(m_{2}^{2}-m_{1}^{2}\right) \rho^{(B Z)}\left(\mathbf{m} ; \eta_{1}, \eta_{2}\right) \mathrm{d} \mathbf{m} .
\end{aligned}
$$

Note that while the Maier-Saupe potential (4) is always true, the Boltzmann distribution (7) is valid only for equilibrium distributions. It is very important to notice this difference between (4) and (7) especially when we consider the free energy of orientational distributions in the stability discussion.

\section{Equilibrium states of a nematic polymer ensemble:}

It has been shown that in the absence of external field, all equilibrium states of nematic polymers are axisymmetric $[8,24,35]$. Without loss of generality, we assume the $m_{3}$-axis is the axis of symmetry. The axisymmetry implies $s_{1}=s_{2}$ and consequently we have $\eta_{2}=0$. Thus, an equilibrium state of nematic polymers is completely specified by $\eta_{1}$.

To facilitate the discussion, we use $r(b)$ to denote the value of $\eta_{1}$ in the equilibrium state(s) corresponding to parameter $b$. More specifically, we adopt the convention that $\eta, \eta_{1}$ and $\eta_{2}$ denote general variables while $r(b)$ denotes specific value of $\eta_{1}$ in the equilibrium state(s).

By definition $r(b)$ is the solution of equation (8). With $\eta_{2}=0$ and $\eta_{1}$ simply denoted by $\eta$, equation (8) becomes

$$
\eta=b \frac{3}{2} \frac{\int_{S}\left(m_{3}^{2}-\frac{1}{3}\right) \exp \left(\eta m_{3}^{2}\right) \mathrm{d} \mathbf{m}}{\int_{S} \exp \left(\eta m_{3}^{2}\right) \mathrm{d} \mathbf{m}} .
$$

It is now convenient to introduce the function $f(\eta)$ defined by

$$
f(\eta) \equiv \frac{3}{2 \eta} \frac{\int_{S}\left(m_{3}^{2}-\frac{1}{3}\right) \exp \left(\eta m_{3}^{2}\right) \mathrm{d} \mathbf{m}}{\int_{S} \exp \left(\eta m_{3}^{2}\right) \mathrm{d} \mathbf{m}} .
$$

Then it follows at once that equation (9) can be written as [35]

$$
\eta\left(\frac{1}{b}-f(\eta)\right)=0
$$

First, observe that $\eta=r_{0}(b)=0$ is always a solution of equation (11), which corresponds to $U(\mathbf{m})=$ constant (independent of $\mathbf{m}$ ), the isotropic equilibrium. Next, anisotropic equilibrium states are the solutions of the equation

$$
f(\eta)=\frac{1}{b}
$$


Using the spherical coordinates,

$$
\begin{aligned}
& m_{1}=\sin \phi \cos \theta, \\
& m_{2}=\sin \phi \sin \theta, \\
& m_{3}=\cos \phi,
\end{aligned}
$$

followed by the substitution $u=\cos \phi$ and then applying integration by parts, we write function $f(\eta)$ as

$$
\begin{aligned}
f(\eta) & =\frac{3}{2 \eta} \frac{\int_{0}^{\pi}\left(\cos ^{2} \phi-\frac{1}{3}\right) \exp \left(\eta \cos ^{2} \phi\right) \sin \phi \mathrm{d} \phi}{\int_{0}^{\pi} \exp \left(\eta \cos ^{2} \phi\right) \sin \phi \mathrm{d} \phi} \\
& =\frac{3}{2 \eta} \frac{\int_{-1}^{1}\left(u^{2}-\frac{1}{3}\right) \exp \left(\eta u^{2}\right) \mathrm{d} u}{\int_{-1}^{1} \exp \left(\eta u^{2}\right) \mathrm{d} u} \\
& =\frac{\int_{-1}^{1}\left(u^{2}-u^{4}\right) \exp \left(\eta u^{2}\right) \mathrm{d} u}{\int_{-1}^{1} \exp \left(\eta u^{2}\right) \mathrm{d} u} \\
& =\frac{\int_{S}\left(m_{3}^{2}-m_{3}^{4}\right) \exp \left(\eta m_{3}^{2}\right) \mathrm{d} \mathbf{m}}{\int_{S} \exp \left(\eta m_{3}^{2}\right) \mathrm{d} \mathbf{m}}
\end{aligned}
$$

In [35], we have shown that the function $f(\eta)$ expressed in (14) satisfies the following properties:

1. $f(0)=\frac{2}{15}$; infinity.

2. $\lim _{\eta \rightarrow+\infty} f(\eta)=0$ and $\lim _{\eta \rightarrow-\infty} f(\eta)=0$; in other words, $f(\eta)$ tends to zero as $|\eta|$ goes to

3. $f(\eta)$ attains its maximum at $\eta^{*}=2.1782879748>0$ where the maximum is $f\left(\eta^{*}\right)=$ $0.14855559992254>0$

4. $f^{\prime}(\eta)>0$ for $\eta<\eta^{*}$ and $f^{\prime}(\eta)<0$ for $\eta>\eta^{*}$.

The graph of the function $f(\eta)$ is depicted in Figure 1.

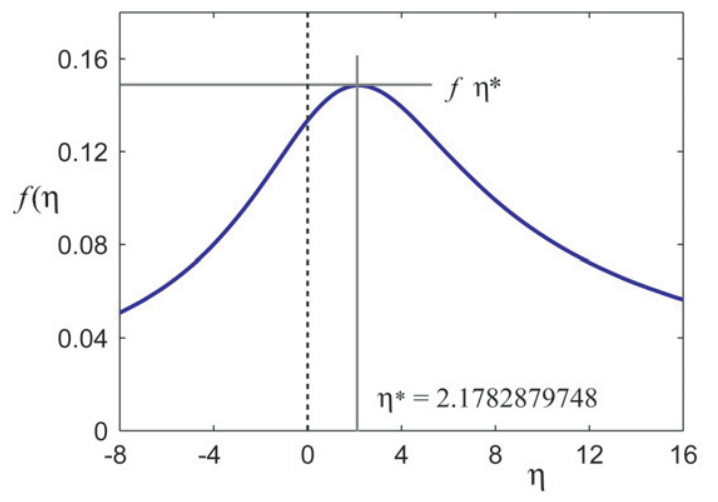

Fig. 1 Graph of the function $f(\eta)$. A key feature of the function $f(\eta)$ is that $f(\eta)$ is strictly increasing for $\eta<\eta^{*}$ (i.e. $f^{\prime}(\eta)>0$ ) and strictly decreasing for $\eta>\eta^{*}$ (i.e., $f^{\prime}(\eta)<0$ )

From these properties of the function $f(\eta)$, we draw conclusions listed below related to equation (12):

- $b^{*}=\frac{1}{f\left(\eta^{*}\right)}=6.7314863965$ is a critical value for parameter $b$. 
- For $b<b^{*}$, equation (12) has no solution; that is, there is no anisotropic state for $b<b^{*}$.

- At $b=b^{*}$, equation (12) has one solution $r\left(b^{*}\right)=\eta^{*}>0$.

- For $b^{*}<b<\frac{15}{2}$, equation (12) attains two solutions: $r_{U}(b)>\eta^{*}>0$ and $0<r_{M}(b)<$ $\eta^{*}$. The equilibrium states corresponding to positive order parameters $r_{U}(b)$ and $r_{M}(b)$ are called prolate states.

- At $b=\frac{15}{2}$, equation (12) possesses two solutions: $r_{U}\left(\frac{2}{15}\right)>\eta^{*}>0$ and $r\left(\frac{2}{15}\right)=0$.

- For $b>\frac{15}{2}$, equation (12) has two solutions: $r_{U}(b)>\eta^{*}>0$ and $r_{L}(b)<0$. The equilibrium state with negative order parameter $r_{L}(b)$ is called oblate state.

Here we use the subscript "U" to refer to the "Upper" part of the phase diagram where $r>\eta^{*}$, the subscript "M" to represent the "Middle" part of the phase diagram where $0<r<\eta^{*}$, and the subscript "L" to denote the "Lower" part of the phase diagram where $r<0$. The phase diagram for nematic polymers is shown in Figure 2.

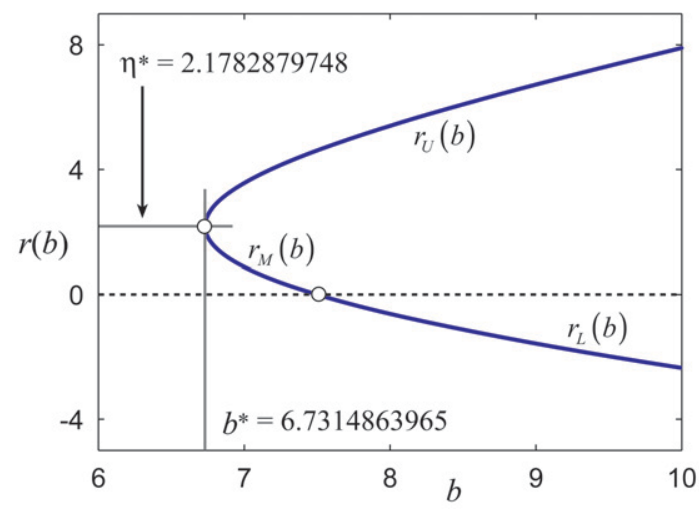

Fig. 2 Phase diagram of nematic polymers

In the above, we have used $b$ as the independent variable and treated $r$ as a function of $b$. However, $r(b)$ is a multi-valued function of $b$ and for $b<b^{*}$ the function $r(b)$ is not even defined. When we study the relation between $b$ and $r$, it is mathematically more convenient if we use $r$ as the independent variable and treat $b$ as a function of $r$ instead. Then, $b(r)$ is a single-valued function of $r$ and is defined for all values of $r$ in $(-\infty,+\infty)$. The function $b(r)$ can be easily determined from equation (12) as

$$
b(r)=\frac{1}{f(r)} .
$$

Once we know the function $b(r)$, the branch $r_{U}(b)$ is simply the inverse function of $b(r)$ for $r>\eta^{*}$; the branch $r_{M}(b)$ is the inverse function of $b(r)$ for $0<r<\eta^{*}$; and the branch $r_{L}(b)$ is the inverse function of $b(r)$ for $r<0$.

\section{Free energy of an orientational distribution:}

The free energy of the orientational distribution $\rho(\mathbf{m})$ is

$$
G([\rho])=\int_{S}\left[\log \rho(\mathbf{m})+\frac{1}{2} U(\mathbf{m},[\rho])\right] \rho(\mathbf{m}) \mathrm{d} \mathbf{m} .
$$

Recall that for any $\rho(\mathbf{m})$, by selecting a proper coordinate system, we can always write the 
Maier-Saupe potential $U(\mathbf{m},[\rho])$ as

$$
U(\mathbf{m},[\rho])=-\eta_{1}\left(m_{3}^{2}-\frac{1}{3}\right)-\eta_{2}\left(m_{2}^{2}-m_{1}^{2}\right),
$$

where $\eta_{1} \equiv b \frac{3}{2}\left(\left\langle m_{3}^{2}\right\rangle-\frac{1}{3}\right)$ and $\eta_{2} \equiv b \frac{1}{2}\left\langle m_{2}^{2}-m_{1}^{2}\right\rangle$. But in general, $\rho(\mathbf{m})$ is not the same as the Boltzmann distribution

$$
\rho(\mathbf{m}) \neq \rho^{(B Z)}\left(\mathbf{m} ; \eta_{1}, \eta_{2}\right) \equiv \frac{\exp \left[\eta_{1} m_{3}^{2}+\eta_{2}\left(m_{2}^{2}-m_{1}^{2}\right)\right]}{\int_{S} \exp \left[\eta_{1} m_{3}^{2}+\eta_{2}\left(m_{2}^{2}-m_{1}^{2}\right)\right] \mathrm{d} \mathbf{m}} .
$$

It is important to point out that even if we restrict our consideration to probability density $\rho(\mathbf{m})$ that has the form of

$$
\rho(\mathbf{m})=\frac{\exp \left[q_{1} m_{3}^{2}+q_{2}\left(m_{2}^{2}-m_{1}^{2}\right)\right]}{\int_{S} \exp \left[q_{1} m_{3}^{2}+q_{2}\left(m_{2}^{2}-m_{1}^{2}\right)\right] \mathrm{d} \mathbf{m}},
$$

we still have $\left(q_{1}, q_{2}\right) \neq\left(\eta_{1}, \eta_{2}\right)$ unless $\rho(\mathbf{m})$ is an equilibrium distribution, which means $q_{2}=0$ and $q_{1}=r(b)$. It is tempting to replace $\rho(\mathbf{m})$ by $\rho^{(B Z)}\left(\mathbf{m} ; \eta_{1}, \eta_{2}\right)$ in (16) and formally define a function of $\left(\eta_{1}, \eta_{2}\right)$

$$
\begin{aligned}
F\left(\eta_{1}, \eta_{2}\right) \equiv & \int_{S}\left[\log \rho^{(B Z)}\left(\mathbf{m} ; \eta_{1}, \eta_{2}\right)+\frac{1}{2}\left(-\eta_{1}\left(m_{3}^{2}-\frac{1}{3}\right)-\eta_{2}\left(m_{2}^{2}-m_{1}^{2}\right)\right)\right] \\
& \times \rho^{(B Z)}\left(\mathbf{m} ; \eta_{1}, \eta_{2}\right) \mathrm{d} \mathbf{m} .
\end{aligned}
$$

Unfortunately, the assertion $F\left(\eta_{1}, \eta_{2}\right)=G([\rho])$ is valid only when $\rho(\mathbf{m})$ is an equilibrium distribution. In other words, at a fixed value of $b$, the assertion $F\left(\eta_{1}, \eta_{2}\right)=G([\rho])$ is true only at a few (at most, three) equilibrium states that are well separated from each other. Therefore, while we can try to analyze the stability of $F\left(\eta_{1}, \eta_{2}\right)$ with respect to perturbations in $\left(\eta_{1}, \eta_{2}\right)$, the stability of $F\left(\eta_{1}, \eta_{2}\right)$ does not inform us about the stability of $G([\rho])$ with respect to perturbations in $\rho(\mathbf{m})$.

\section{Stability Analysis}

In this section we present a detailed stability analysis on the equilibria.

To make our presentation easy to follow, we divide our approach into five steps. In the first step, we minimize the free energy functional $G([\rho])$ over a set $B$ of functions with the second moments fixed, specified by two parameters $\left(\eta_{1}, \eta_{2}\right)$. The resulting free energy is a function of two variables $G\left(\eta_{1}, \eta_{2}\right)$. The stability of $G([\rho])$ is equivalent to the stability of $G\left(\eta_{1}, \eta_{2}\right)$. In the second step, we show that the minimum of $G([\rho])$ is attained at a probability density $\rho^{*}$ of the Boltzmann form. The coefficients $\left(q_{1}, q_{2}\right)$ in the exponent of $\rho^{*}$ define a one-to-one mapping between $\left(\eta_{1}, \eta_{2}\right)$ and $\left(q_{1}, q_{2}\right)$. Thus, we can rewrite $G\left(\eta_{1}, \eta_{2}\right)$ as $H\left(q_{1}, q_{2}\right)$. The important point to note here is that $\left(q_{1}, q_{2}\right)$ is in general different from $\left(\eta_{1}, \eta_{2}\right)$ unless $\rho^{*}$ is an equilibrium state, which is an improper assumption in stability analysis. In the third step, we compute the Hessian matrix of $H\left(q_{1}, q_{2}\right)$ with respect to $\left(q_{1}, q_{2}\right)$, which is fortunately a diagonal matrix. In the fourth step, we consider the stability of the isotropic equilibrium state. At the last step we focus our attention on the stability of the anisotropic equilibrium states. 
Step 1 We consider the constrained minimum over the set

$$
B\left(\eta_{1}, \eta_{2}\right)=\left\{\rho(\mathbf{m}) \mid\left(\left\langle m_{3}^{2}\right\rangle_{[\rho]}-\frac{1}{3}\right)=\frac{2}{3 b} \eta_{1},\left\langle m_{2}^{2}-m_{1}^{2}\right\rangle_{[\rho]}=\frac{2}{b} \eta_{2}\right\},
$$

where the probability density $\rho(\mathbf{m})$ is involved in the average in the following way:

$$
\langle f(\mathbf{m})\rangle_{[\rho]} \equiv \int_{S} f(\mathbf{m}) \rho(\mathbf{m}) \mathrm{d} \mathbf{m} .
$$

The minimum of $G([\rho])$ over the set $B\left(\eta_{1}, \eta_{2}\right)$ is a function of $\left(\eta_{1}, \eta_{2}\right)$ :

$$
G\left(\eta_{1}, \eta_{2}\right) \equiv \min _{\rho(\mathbf{m}) \in B\left(\eta_{1}, \eta_{2}\right)} G([\rho])
$$

From the definition of $G\left(\eta_{1}, \eta_{2}\right)$, we see that the stability of $G([\rho])$ with respect to $\rho$ is the same as the stability of $G\left(\eta_{1}, \eta_{2}\right)$ with respect to $\left(\eta_{1}, \eta_{2}\right)$.

Step 2 We study where $G([\rho])$ attains the minimum over the set $B\left(\eta_{1}, \eta_{2}\right)$ and use the result to calculate $G\left(\eta_{1}, \eta_{2}\right)$. For $\rho(\mathbf{m}) \in B\left(\eta_{1}, \eta_{2}\right)$, we have

$$
\begin{aligned}
U(\mathbf{m},[\rho]) & =-\eta_{1}\left(m_{3}^{2}-\frac{1}{3}\right)-\eta_{2}\left(m_{2}^{2}-m_{1}^{2}\right) \\
\frac{1}{2} \int_{S} U(\mathbf{m},[\rho]) \rho(\mathbf{m}) \mathrm{d} \mathbf{m} & =\frac{1}{2}\left(-\eta_{1}\left\langle m_{3}^{2}-\frac{1}{3}\right\rangle_{[\rho]}-\eta_{2}\left\langle m_{2}^{2}-m_{1}^{2}\right\rangle_{[\rho]}\right) \\
& =-\left(\frac{1}{3 b} \eta_{1}^{2}+\frac{1}{b} \eta_{2}^{2}\right) .
\end{aligned}
$$

That is, the second term in the free energy $G([\rho])$ given in (16) is actually a constant over the set $B\left(\eta_{1}, \eta_{2}\right)$. It follows that we only need to look at the first term of $G([\rho])$ in the constrained minimization problem. Let us denote the first term by

$$
G_{1}([\rho]) \equiv \int_{S} \log \rho(\mathbf{m}) \rho(\mathbf{m}) \mathrm{d} \mathbf{m} .
$$

The constrained minimization problem then becomes

$$
\underset{\rho(\mathbf{m}) \in B\left(\eta_{1}, \eta_{2}\right)}{\operatorname{argmin}} G_{1}([\rho])
$$

Suppose the constrained minimum is attained at $\rho^{*}(\mathbf{m})$. We consider the perturbed probability density $\rho(\mathbf{m})=\rho^{*}(\mathbf{m})+s \Delta \rho(\mathbf{m})$ where $\Delta \rho(\mathbf{m})$ satisfies the following properties:

$$
\begin{aligned}
& \int_{S} \Delta \rho(\mathbf{m}) \mathrm{d} \mathbf{m}=0, \\
& \int_{S}\left(m_{3}^{2}-\frac{1}{3}\right) \Delta \rho(\mathbf{m}) \mathrm{d} \mathbf{m}=0, \\
& \int_{S}\left(m_{2}^{2}-m_{1}^{2}\right) \Delta \rho(\mathbf{m}) \mathrm{d} \mathbf{m}=0 .
\end{aligned}
$$

$G_{1}\left(\left[\rho^{*}+s \Delta \rho\right]\right)$ attaining the minimum at $s=0$ implies $\left.\frac{\mathrm{d} G_{1}\left(\left[\rho^{*}+s \Delta \rho\right]\right)}{\mathrm{d} s}\right|_{s=0}=0$, which gives us

$$
\int_{S} \log \rho^{*}(\mathbf{m}) \Delta \rho(\mathbf{m}) \mathrm{d} \mathbf{m}=0
$$


Since equation (20) is true for all $\Delta \rho(\mathbf{m})$ satisfying (19), it implies that

$$
\log \left(\underset{\rho(\mathbf{m}) \in B\left(\eta_{1}, \eta_{2}\right)}{\operatorname{argmin}} G_{1}([\rho])\right)=\log \rho^{*}(\mathbf{m})=q_{0}+q_{1}\left(m_{3}^{2}-\frac{1}{3}\right)+q_{2}\left(m_{2}^{2}-m_{1}^{2}\right) .
$$

The result of the constrained minimization given in (21) defines a mapping from $\left(\eta_{1}, \eta_{2}\right)$ to $\left(q_{1}, q_{2}\right)$. The mapping from $\left(\eta_{1}, \eta_{2}\right)$ to $\left(q_{1}, q_{2}\right)$ is not expressed in an explicit form and it is not immediately obvious if the mapping is single-valued. However, the inverse mapping from $\left(q_{1}, q_{2}\right)$ back to $\left(\eta_{1}, \eta_{2}\right)$ is single-valued and has a simple explicit expression:

$$
\begin{aligned}
& \eta_{1}=b \frac{3}{2}\left\langle m_{3}^{2}-\frac{1}{3}\right\rangle, \\
& \eta_{2}=b \frac{1}{2}\left\langle m_{2}^{2}-m_{1}^{2}\right\rangle,
\end{aligned}
$$

where the average is with respect to the probability density $\rho^{*}\left(\mathbf{m}, q_{1}, q_{2}\right)$ given by

$$
\begin{aligned}
\rho^{*}\left(\mathbf{m}, q_{1}, q_{2}\right) & \equiv \frac{1}{Z\left(q_{1}, q_{2}\right)} \exp \left[q_{1}\left(m_{3}^{2}-\frac{1}{3}\right)+q_{2}\left(m_{2}^{2}-m_{1}^{2}\right)\right], \\
Z\left(q_{1}, q_{2}\right) & \equiv \int_{S} \exp \left[q_{1}\left(m_{3}^{2}-\frac{1}{3}\right)+q_{2}\left(m_{2}^{2}-m_{1}^{2}\right)\right] \mathrm{d} \mathbf{m} .
\end{aligned}
$$

To prepare for the computation of the Hessian matrix, we prove the following lemma, which asserts that the mapping from $\left(\eta_{1}, \eta_{2}\right)$ to $\left(q_{1}, q_{2}\right)$ is also single-valued.

Lemma 1 For any fixed $\left(\eta_{1}, \eta_{2}\right)$, there is only one set of $\left(q_{1}, q_{2}\right)$ satisfying (22).

Proof We prove by contradiction. To accomplish this, suppose $\left(q_{1}^{(1)}, q_{2}^{(1)}\right)$ and $\left(q_{1}^{(2)}, q_{2}^{(2)}\right)$ are different from each other, and both satisfy (22).

Let $\rho^{*}(\mathbf{m}) \equiv \rho^{*}\left(\mathbf{m}, q_{1}^{(1)}, q_{2}^{(1)}\right)$ and $\Delta \rho(\mathbf{m}) \equiv \rho^{*}\left(\mathbf{m}, q_{1}^{(2)}, q_{2}^{(2)}\right)-\rho^{*}\left(\mathbf{m}, q_{1}^{(1)}, q_{2}^{(1)}\right)$.

We calculate the first and second derivatives of the function $G_{1}\left(\left[\rho^{*}+s \Delta \rho\right]\right)$ with respect to $s$ as follows:

$$
\begin{aligned}
& \frac{\mathrm{d} G_{1}\left(\left[\rho^{*}+s \Delta \rho\right]\right)}{\mathrm{d} s}=\int_{S} \log \left(\rho^{*}+s \Delta \rho\right) \Delta \rho \mathrm{d} \mathbf{m}, \\
& \frac{\mathrm{d}^{2} G_{1}\left(\left[\rho^{*}+s \Delta \rho\right]\right)}{\mathrm{d} s^{2}}=\int_{S} \frac{1}{\left(\rho^{*}+s \Delta \rho\right)}(\Delta \rho)^{2} \mathrm{~d} \mathbf{m}>0 .
\end{aligned}
$$

Since both $\left(q_{1}^{(1)}, q_{2}^{(1)}\right)$ and $\left(q_{1}^{(2)}, q_{2}^{(2)}\right)$ satisfy (22), $\Delta \rho(\mathbf{m})$ must satisfy (19), which means

$$
\left.\frac{\mathrm{d} G_{1}\left(\left[\rho^{*}+s \Delta \rho\right]\right)}{\mathrm{d} s}\right|_{s=0}=\int_{S} \log \left(\rho^{*}\right) \Delta \rho \mathrm{d} \mathbf{m}=0
$$

Taylor's theorem tells us

$$
G_{1}\left(\left[\rho^{*}+\Delta \rho\right]\right)=G_{1}\left(\left[\rho^{*}\right]\right)+\left.\frac{1}{2} \frac{\mathrm{d}^{2} G_{1}\left(\left[\rho^{*}+s \Delta \rho\right]\right)}{\mathrm{d} s^{2}}\right|_{s=\xi}>G_{1}\left(\left[\rho^{*}\right]\right),
$$

which is

$$
G_{1}\left(\left[\rho^{*}\left(\mathbf{m}, q_{1}^{(2)}, q_{2}^{(2)}\right)\right]\right)>G_{1}\left(\left[\rho^{*}\left(\mathbf{m}, q_{1}^{(1)}, q_{2}^{(1)}\right)\right]\right) .
$$

Repeating the argument with the roles of $\left(q_{1}^{(1)}, q_{2}^{(1)}\right)$ and $\left(q_{1}^{(2)}, q_{2}^{(2)}\right)$ swapped, we get

$$
G_{1}\left(\left[\rho^{*}\left(\mathbf{m}, q_{1}^{(1)}, q_{2}^{(1)}\right)\right]\right)>G_{1}\left(\left[\rho^{*}\left(\mathbf{m}, q_{1}^{(2)}, q_{2}^{(2)}\right)\right]\right),
$$


which contradicts with (25). Thus the claim is established: $\left(q_{1}^{(1)}, q_{2}^{(1)}\right)$ and $\left(q_{1}^{(2)}, q_{2}^{(2)}\right)$ must be the same.

We conclude that the mapping between $\left(\eta_{1}, \eta_{2}\right)$ and $\left(q_{1}, q_{2}\right)$ is one-to-one. As a result, we can express the minimum free energy over the set $B\left(\eta_{1}, \eta_{2}\right)$ as a function of $\left(q_{1}, q_{2}\right)$ :

$$
\begin{aligned}
H\left(q_{1}, q_{2}\right) & \equiv \min _{\rho(\mathbf{m}) \in B\left(\eta_{1}, \eta_{2}\right)} G([\rho]) \\
& =q_{1}\left\langle m_{3}^{2}-\frac{1}{3}\right\rangle+q_{2}\left\langle m_{2}^{2}-m_{1}^{2}\right\rangle-\log \left(Z\left(q_{1}, q_{2}\right)\right)-\frac{1}{b}\left(\frac{1}{3} \eta_{1}^{2}+\eta_{2}^{2}\right) \\
& =\frac{2}{b}\left(\frac{1}{3} q_{1} \eta_{1}+q_{2} \eta_{2}\right)-\log \left(Z\left(q_{1}, q_{2}\right)\right)-\frac{1}{b}\left(\frac{1}{3} \eta_{1}^{2}+\eta_{2}^{2}\right),
\end{aligned}
$$

where the mapping from $\left(q_{1}, q_{2}\right)$ to $\left(\eta_{1}, \eta_{2}\right)$ is given in (22) and equations (16), (23) and (24) have been invoked.

The stability of $G([\rho])$ with respect to $\rho(\mathbf{m})$ is the same as the stability of $H\left(q_{1}, q_{2}\right)$ with respect to $\left(q_{1}, q_{2}\right)$, which is determined by its Hessian matrix. The Hessian matrix (or simply the Hessian) is just the square matrix of second-order partial derivatives of a function. It can be used to determine the stability or instability of a system.

Step 3 We now calculate the Hessian matrix of $H\left(q_{1}, q_{2}\right)$. To do so, we begin by differentiating $\eta_{1}, \eta_{2}$ and $\log \left(Z\left(q_{1}, q_{2}\right)\right)$ with respect to $q_{1}$ and $q_{2}$. Referring to (22), (23) and (24) and by direct computation, we obtain the following partial derivatives:

$$
\begin{aligned}
\frac{\partial \rho^{*}\left(\mathbf{m}, q_{1}, q_{2}\right)}{\partial q_{1}} & =\left(m_{3}^{2}-\frac{1}{3}\right) \rho^{*}\left(\mathbf{m}, q_{1}, q_{2}\right)-\left\langle m_{3}^{2}-\frac{1}{3}\right\rangle \rho^{*}\left(\mathbf{m}, q_{1}, q_{2}\right) \\
& =\left(m_{3}^{2}-\left\langle m_{3}^{2}\right\rangle\right) \rho^{*}\left(\mathbf{m}, q_{1}, q_{2}\right) \\
\frac{\partial \rho^{*}\left(\mathbf{m}, q_{1}, q_{2}\right)}{\partial q_{2}} & =\left(\left(m_{2}^{2}-m_{1}^{2}\right)-\left\langle m_{2}^{2}-m_{1}^{2}\right\rangle\right) \rho^{*}\left(\mathbf{m}, q_{1}, q_{2}\right) \\
\frac{\partial \eta_{1}}{\partial q_{1}} & =b \frac{3}{2}\left(\left\langle m_{3}^{4}\right\rangle-\left\langle m_{3}^{2}\right\rangle^{2}\right)>0 \\
\frac{\partial \eta_{1}}{\partial q_{2}} & =b \frac{3}{2}\left(\left\langle m_{3}^{2}\left(m_{2}^{2}-m_{1}^{2}\right)\right\rangle-\left\langle m_{3}^{2}\right\rangle\left\langle m_{2}^{2}-m_{1}^{2}\right\rangle\right) \\
\frac{\partial \eta_{2}}{\partial q_{1}} & =b \frac{1}{2}\left(\left\langle m_{3}^{2}\left(m_{2}^{2}-m_{1}^{2}\right)\right\rangle-\left\langle m_{3}^{2}\right\rangle\left\langle m_{2}^{2}-m_{1}^{2}\right\rangle\right) \\
\frac{\partial \eta_{2}}{\partial q_{2}} & =b \frac{1}{2}\left(\left\langle\left(m_{2}^{2}-m_{1}^{2}\right)^{2}\right\rangle-\left\langle m_{2}^{2}-m_{1}^{2}\right\rangle^{2}\right)>0 \\
\frac{\partial \log \left(Z\left(q_{1}, q_{2}\right)\right)}{\partial q_{1}} & =\left\langle m_{3}^{2}-\frac{1}{3}\right\rangle=\frac{2}{3 b} \eta_{1} \\
\frac{\partial \log \left(Z\left(q_{1}, q_{2}\right)\right)}{\partial q_{2}} & =\left\langle m_{2}^{2}-m_{1}^{2}\right\rangle=\frac{2}{b} \eta_{2} .
\end{aligned}
$$

Applying these results to differentiate $H\left(q_{1}, q_{2}\right)$ given in (27), we get

$$
\begin{aligned}
& \frac{\partial H\left(q_{1}, q_{2}\right)}{\partial q_{1}}=\frac{2}{b}\left(\frac{1}{3}\left(q_{1}-\eta_{1}\right) \frac{\partial \eta_{1}}{\partial q_{1}}+\left(q_{2}-\eta_{2}\right) \frac{\partial \eta_{2}}{\partial q_{1}}\right), \\
& \frac{\partial H\left(q_{1}, q_{2}\right)}{\partial q_{2}}=\frac{2}{b}\left(\frac{1}{3}\left(q_{1}-\eta_{1}\right) \frac{\partial \eta_{1}}{\partial q_{2}}+\left(q_{2}-\eta_{2}\right) \frac{\partial \eta_{2}}{\partial q_{2}}\right) .
\end{aligned}
$$


To determine the stability of an equilibrium state, we only need to calculate the Hessian matrix of $H\left(q_{1}, q_{2}\right)$ evaluated at the equilibrium state.

At an equilibrium state, $\left.\left(\eta_{1}, \eta_{2}\right)\right|_{e q}$ satisfies equation (8). The mapping between $\left(q_{1}, q_{2}\right)$ and $\left(\eta_{1}, \eta_{2}\right)$ is described in (22). Comparing (8) and (22), we conclude

$$
\left.\left(q_{1}, q_{2}\right)\right|_{e q}=\left.\left(\eta_{1}, \eta_{2}\right)\right|_{e q} .
$$

In addition, all equilibrium states are axisymmetric, which amounts to

$$
\left.q_{2}\right|_{e q}=0 .
$$

Thus, at an equilibrium state, we have

$$
\begin{array}{r}
\left.\left\langle m_{2}^{2}-m_{1}^{2}\right\rangle\right|_{e q}=0, \\
\left.\left\langle m_{3}^{2}\left(m_{2}^{2}-m_{1}^{2}\right)\right\rangle\right|_{e q}=0,
\end{array}
$$

which leads to

$$
\begin{aligned}
& \left.\frac{\partial \eta_{1}}{\partial q_{2}}\right|_{e q}=0 \\
& \left.\frac{\partial \eta_{2}}{\partial q_{1}}\right|_{e q}=0 .
\end{aligned}
$$

Calculating the Hessian matrix of $H\left(q_{1}, q_{2}\right)$ and evaluating it at the equilibrium state, we obtain

$$
\begin{aligned}
& \left.\frac{\partial^{2} H\left(q_{1}, q_{2}\right)}{\partial q_{1}^{2}}\right|_{e q}=\left.\frac{2}{3 b}\left(1-\frac{\partial \eta_{1}}{\partial q_{1}}\right) \frac{\partial \eta_{1}}{\partial q_{1}}\right|_{e q}, \\
& \left.\frac{\partial^{2} H\left(q_{1}, q_{2}\right)}{\partial q_{1} \partial q_{2}}\right|_{e q}=0 \\
& \left.\frac{\partial^{2} H\left(q_{1}, q_{2}\right)}{\partial q_{2}^{2}}\right|_{e q}=\left.\frac{2}{b}\left(1-\frac{\partial \eta_{2}}{\partial q_{2}}\right) \frac{\partial \eta_{2}}{\partial q_{2}}\right|_{e q} .
\end{aligned}
$$

Note that both $\frac{\partial \eta_{1}}{\partial q_{1}}$ and $\frac{\partial \eta_{2}}{\partial q_{2}}$ are positive. Consequently, the stability of $H\left(q_{1}, q_{2}\right)$ is determined by the two quantities below:

$$
\begin{aligned}
& H_{11} \equiv 1-\left.\frac{\partial \eta_{1}}{\partial q_{1}}\right|_{e q}=1-\left.b \frac{3}{2}\left(\left\langle m_{3}^{4}\right\rangle-\left\langle m_{3}^{2}\right\rangle^{2}\right)\right|_{e q}, \\
& H_{22} \equiv 1-\left.\frac{\partial \eta_{2}}{\partial q_{2}}\right|_{e q}=1-\left.b \frac{1}{2}\left\langle\left(m_{2}^{2}-m_{1}^{2}\right)^{2}\right\rangle\right|_{e q} .
\end{aligned}
$$

Since $\left.q_{2}\right|_{e q}=0$, we can write $\left.\left\langle\left(m_{2}^{2}-m_{1}^{2}\right)^{2}\right\rangle\right|_{e q}$ as

$$
\begin{aligned}
\left.\left\langle\left(m_{2}^{2}-m_{1}^{2}\right)^{2}\right\rangle\right|_{e q} & =\frac{\int_{S}\left(m_{2}^{2}-m_{1}^{2}\right)^{2} \exp \left(q_{1} m_{3}^{2}\right) \mathrm{d} \mathbf{m}}{\int_{S} \exp \left(q_{1} m_{3}^{2}\right) \mathrm{d} \mathbf{m}} \\
& =\frac{\int_{0}^{\pi}\left(\sin ^{2} \phi\right)^{2}\left(\frac{1}{2 \pi} \int_{0}^{2 \pi}\left(\cos ^{2} \theta-\sin ^{2} \theta\right)^{2} d \theta\right) \exp \left(q_{1} \cos ^{2} \phi\right) \sin \phi \mathrm{d} \phi}{\int_{0}^{\pi} \exp \left(q_{1} \cos ^{2} \phi\right) \sin \phi \mathrm{d} \phi} \\
& =\left.\frac{1}{2}\left\langle\left(1-m_{3}^{2}\right)^{2}\right\rangle\right|_{e q} .
\end{aligned}
$$


Thus, we write $H_{22}$ as

$$
H_{22}=1-\left.b \frac{1}{4}\left\langle\left(1-m_{3}^{2}\right)^{2}\right\rangle\right|_{e q} .
$$

Step 4 Now we turn our attention to the stability of the isotropic state, which is described by $\left.\left(q_{1}, q_{2}\right)\right|_{e q}=(0,0)$. With the help of the spherical coordinates $(13)$, it is straightforward to derive

$$
\begin{aligned}
\left.\left\langle m_{3}^{2}\right\rangle\right|_{e q} & =\frac{\int_{0}^{\pi} \cos ^{2} \phi \sin \phi \mathrm{d} \phi}{\int_{0}^{\pi} \sin \phi \mathrm{d} \phi}=\int_{0}^{1} u^{2} \mathrm{~d} u=\frac{1}{3} \\
\left.\left\langle m_{3}^{4}\right\rangle\right|_{e q} & =\int_{0}^{1} u^{4} \mathrm{~d} u=\frac{1}{5} \\
\left.\left\langle\left(1-m_{3}^{2}\right)^{2}\right\rangle\right|_{e q} & =\int_{0}^{1}\left(1-u^{2}\right)^{2} \mathrm{~d} u=\frac{8}{15} \\
H_{11} & =1-b \frac{2}{15} \\
H_{22} & =1-b \frac{2}{15} .
\end{aligned}
$$

It follows readily that the Hessian matrix evaluated at the isotropic equilibrium state is positive definite if $b<\frac{15}{2}$ and negative definite if $b>\frac{15}{2}$. Therefore, the isotropic equilibrium state is stable for $b<\frac{15}{2}$ whereas it is unstable for $b>\frac{15}{2}$.

Step 5 Finally, we close our analysis by addressing the stability of anisotropic equilibrium states. An anisotropic equilibrium state is described by $q_{1}=r(b)$ and $q_{2}=0$. First we look at $H_{11}$. We use the definition of function $f(\eta)$ given in (10) to write

$$
q f(q)=\frac{3}{2} \frac{\int_{S}\left(m_{3}^{2}-\frac{1}{3}\right) \exp \left(q m_{3}^{2}\right) \mathrm{d} \mathbf{m}}{\int_{S} \exp \left(q m_{3}^{2}\right) \mathrm{d} \mathbf{m}}=\left.\frac{3}{2}\left\langle m_{3}^{2}-\frac{1}{3}\right\rangle\right|_{\left(q_{1}, q_{2}\right)=(q, 0)} .
$$

Note that (28) is true for all values of $q$, not just at the equilibrium state $q=r(b)$. Differentiating (28) with respect to $q$ and then evaluating it at the equilibrium state, we have

$$
f(r(b))+r(b) f^{\prime}(r(b))=\left.\frac{3}{2}\left(\left\langle m_{3}^{4}\right\rangle-\left\langle m_{3}^{2}\right\rangle^{2}\right)\right|_{e q} .
$$

Since $r(b)$ is the solution of equation (12), it satisfies $f(r(b))=1 / b$. Substituting this into (29) yields

$$
H_{11}=1-\left.b \frac{3}{2}\left(\left\langle m_{3}^{4}\right\rangle-\left\langle m_{3}^{2}\right\rangle^{2}\right)\right|_{e q}=-b r(b) f^{\prime}(r(b)) .
$$

Recall that in [35] we have derived that

$$
f^{\prime}(\eta)<0 \text { for } \eta>\eta^{*} \text { and } f^{\prime}(\eta)>0 \text { for } \eta<\eta^{*}
$$

and recall the classification of three anisotropic branches

$$
r_{U}(b)>\eta^{*}, \quad 0<r_{M}(b)<\eta^{*}, \quad \text { and } \quad r_{L}(b)<0,
$$

we see that

$$
H_{11}=\left\{\begin{array}{l}
>0 \text { for } r_{U}(b) \\
<0 \text { for } r_{M}(b) \\
>0 \text { for } r_{L}(b)
\end{array}\right.
$$


Next we examine $H_{22}$. Multiplying $H_{22}$ by $4 / b$, using $1 / b=f(r(b))$ and the expression of $f(\eta)$ given in (14), we have

$$
\begin{aligned}
\frac{4}{b} H_{22} & =4 f(r(b))-\left.\left\langle\left(1-m_{3}^{2}\right)^{2}\right\rangle\right|_{\left(q_{1}, q_{2}\right)=(r(b), 0)} \\
& =\left.4\left\langle m_{3}^{2}-m_{3}^{4}\right\rangle\right|_{\left(q_{1}, q_{2}\right)=(r(b), 0)}-\left.\left\langle\left(1-m_{3}^{2}\right)^{2}\right\rangle\right|_{\left(q_{1}, q_{2}\right)=(r(b), 0)} \\
& =\left.\left\langle\left(1-m_{3}^{2}\right)\left(5 m_{3}^{2}-1\right)\right\rangle\right|_{\left(q_{1}, q_{2}\right)=(r(b), 0)} \equiv h(r(b)),
\end{aligned}
$$

where the function $h(q)$ is defined as

$$
\left.h(q) \equiv\left\langle\left(1-m_{3}^{2}\right)\left(5 m_{3}^{2}-1\right)\right\rangle\right|_{\left(q_{1}, q_{2}\right)=(q, 0)} .
$$

To determine the sign of $h(r(b))$, we study the behavior of function $h(q)$. We start by verifying

$$
h(0)=\int_{0}^{1}\left(1-u^{2}\right)\left(5 u^{2}-1\right) \mathrm{d} u=0 .
$$

Next we differentiate $h(q)$ :

$$
\begin{aligned}
h^{\prime}(q) & =\left.\left\langle\left(1-m_{3}^{2}\right)\left(5 m_{3}^{2}-1\right)\left(m_{3}^{2}-\left\langle m_{3}^{2}\right\rangle\right)\right\rangle\right|_{\left(q_{1}, q_{2}\right)=(q, 0)} \\
& =\left.\frac{1}{5}\left\langle\left(1-m_{3}^{2}\right)\left(5 m_{3}^{2}-1\right)\left(5 m_{3}^{2}-1+1-5\left\langle m_{3}^{2}\right\rangle\right)\right\rangle\right|_{\left(q_{1}, q_{2}\right)=(q, 0)} \\
& =\left.\frac{1}{5}\left\langle\left(1-m_{3}^{2}\right)\left(5 m_{3}^{2}-1\right)^{2}\right\rangle\right|_{\left(q_{1}, q_{2}\right)=(q, 0)}+\frac{1}{5}\left(1-5\left\langle m_{3}^{2}\right\rangle\right) h(q) .
\end{aligned}
$$

Note that the first term in $h^{\prime}(q)$ is always positive for all values of $q$ :

$$
\left.\left\langle\left(1-m_{3}^{2}\right)\left(5 m_{3}^{2}-1\right)^{2}\right\rangle\right|_{\left(q_{1}, q_{2}\right)=(q, 0)}>0 .
$$

As a result, the function $h(q)$ has the property that $h^{\prime}(q)>0$ wherever $h(q)=0$. This property along with $h(0)=0$ implies

$$
h(q)>0 \text { for } q>0 \text { and } h(q)<0 \text { for } q<0 .
$$

Thus, for $H_{22}$, we have

$$
H_{22}=\frac{b}{4} h(r(b))= \begin{cases}>0 & \text { for } r_{U}(b), \\ >0 & \text { for } r_{M}(b), \\ <0 & \text { for } r_{L}(b) .\end{cases}
$$

Putting the results from (30) and (33) together, we arrive at

$$
\left(H_{11}, H_{22}\right)= \begin{cases}(+,+) & \text { for } r_{U}(b), \\ (-,+) & \text { for } r_{M}(b), \\ (+,-) & \text { for } r_{L}(b) .\end{cases}
$$

Therefore, only the highly aligned prolate branch $r_{U}(b)$ is stable while the weakly aligned prolate branch $r_{M}(b)$ and the oblate branch $r_{L}(b)$ are unstable. More specifically, the branch $r_{U}(b)$ is stable to all perturbations; the branch $r_{M}(b)$ is unstable with respect to perturbation in $q_{1}$, i.e., axisymmetric perturbation along the axis of symmetry. Such perturbation will drive the system either to highly aligned prolate branch $r_{U}(b)$ or the isotropic branch. In contrast, the oblate branch $r_{L}(b)$ is unstable with respect to perturbation in $q_{2}$, i.e., perturbation away from axisymmetry. This kind of perturbation will carry the system to the prolate state. 


\section{Conclusions and Perspective Views}

The stability analysis performed in this study confirms the well-known hysteresis phenomenon of the equilibria of the nematic liquid crystalline polymers governed by the Smoluchowski equation with the Maier-Saupe intermolecular potential. In particular, the isotropic equilibrium state is stable for $b<15 / 2$ and it becomes unstable for $b>15 / 2$ where $b$ is proportional to the normalized polymer concentration and it describes the strength of the intermolecular interactions. As for the anisotropic equilibrium states, only the highly aligned prolate state is stable while the less aligned prolate state $\left(b^{*}<b<15 / 2\right)$ and the oblate state $(b>15 / 2)$ are unstable. The anisotropic states occur when $b>b^{*}$ where the critical point $b^{*}=6.7314863965$.

While the mathematical study on the Smoluchowski equation with the Maier-Saupe potential is getting well-developed, rigorous proofs on the similiar results for the Smoluchowski equation with the Onsager potential remain to be explored. We end this paper by adopting a perspective view provided by Donald, Windle and Hanna [5]: "It is clear that 'self-assembly', where molecules are designed so that they organise themselves into larger scale structures in order to achieve special properties, will be a significant objective in materials science in the twenty-first century. The demands of nanotechnology require an ever finer control over the molecular arrangements within new materials. The fact that liquid crystallinity itself is a form of orientational self-assembly, coupled with the fact that the molecules in a mesophase can be steered by external fields, means that the principles undering the science of LCPs can only grow in significance in the years ahead."

\section{References}

[1] Bird B, Armstrong R C, Hassager O. Dynamics of Polymeric Liquids, Vol 1. Wiley, 1987

[2] Constantin P, Kevrekidis I, Titi E S. Asymptotic states of a Smoluchowski equation. Arch Rat Mech Anal, 2004, 174: 365-384

[3] Constantin P, Kevrekidis I, Titi E S. Remarks on a Smoluchowski equation. Discrete and Continuous Dynamical Systems, 2004, 11: 101-112

[4] Constantin P, Vukadinovic J. Note on the number of steady states for a 2D Smoluchowski equation. Nonlinearity, 2005, 18: 441-443

[5] Donald A M, Windle A H, Hanna S. Liquid Crystalline Polymers. 2nd ed. Cambridge University Press, 2006

[6] Doi M, Edwards S F. The Theory of Polymer Dynamics. Oxford University Press, 1986

[7] Ericksen J L. Equilibrium theory of liquid crystals//Brown G H. Advances in Liquid Crystals. New York: Academic, 1976: 233-298

[8] Fatkullin I, Slastikov V. Critical points of the Onsager functional on a sphere. Nonlinearity, 2005, 18: $2565-2580$

[9] Flory P J. Statistical thermodynamics of semi-flexible chain molecules. Proc R Soc Lond A, 1956, 234: 60-73

[10] Flory P J. Molecular theory of liquid crystals. Adv Polym Sci, 1984, 59: 1-36

[11] Forest M G, Zhou R, Wang Q. Symmetries of the Doi kinetic theory for nematic polymers of arbitrary aspect ratio: at rest and in linear flows. Phys Rev E, 2002, 66: 031712

[12] Forest M G, Wang Q, Zhou R. The flow-phase diagram of Doi-Hess theory for sheared nematic polymers II: finite shear rates. Rheol Acta, 2004, 44(1): 80-93

[13] Forest M G, Zhou R, Wang Q. The weak shear phase diagram for nematic polymers. Rheol Acta, 2004, 43(1): $17-37$ 
[14] Forest M G, Zhou R, Wang Q. Chaotic boundaries of nematic polymers in mixed shear and extensional flows. Phys Rev Lett, 2004, 93(8): 088301-088305

[15] Forest M G, Zhou R, Wang Q. Kinetic structure simulations of nematic polymers in plane Couette cells, I: The algorithm and benchmarks. Multiscale Model Sim, 2005, 3(4): 853-870

[16] Forest M G, Zhou R, Wang Q. Scaling behavior of kinetic orientational distributions for dilute nematic polymers in weak shear. J Non-Newtonian Fluid Mech, 2004, 116: 183-204

[17] Forest M G, Wang Q. Monodomain response of finite-aspect-ratio macromolecules in shear and related linear flows. Rheol Acta, 2003, 42: 20-46

[18] De Gennes P G, Prost J. The Physics of Liquid Crystals. 2nd ed. Oxford: Claredon Press, 1993

[19] Hess S Z. Fokker-Planck-equation approach to flow alignment in liquid crystals. Z Naturforsch A, 1976, 31A: 1034-1037

[20] Hess S, Kroger M. Regular and chaotic orientational and rheological behaviour of liquid crystals. J Phys: Condens Matter, 2004, 16: S3835-S3859

[21] Ji G, Wang Q, Zhang P, Zhou H. Study of phase transition in homogeneous, rigid extended nematics and magnetic suspensions using an order-reduction method. Phys Fluid, 2006, 18: 123103

[22] Landau L D. Collected Papers of L.D. Landau, edited by D. ter Haar. New York: Gordon and Breach, 1965

[23] Leslie F M. Theory of flow phenomena in liquid crystals//Brown G H. Advances in Liquid Crystals. New York: Adademic, 1979

[24] Liu H, Zhang H, Zhang P. Axial symmetry and classification of stationary solutions of Doi-Onsager equation on the sphere with Maier-Saupe potential. Comm Math Sci, 2005, 3: 201-218

[25] Luo C, Zhang H, Zhang P. The structure of equilibrium solution of 1D Smoluchowski equation. Nonlinearity, 2005, 18: 379-389

[26] Maier W, Saupe A Z. Eine einfache molekulare Theorie des nematischen kristallinflussigen Zustandes. A Z Naturforsc, 1958, 13A: 564-566

[27] Maier W, Saupe A Z. Eine einfache molekular-statistische Theorie der nematischen Kristallinflussigen Phase 1. A Z Naturforsc, 1959, 14A: 882-889

[28] Maier W, Saupe A Z. Eine einfache molekular-statistische Theorie der nematischen Kristallinflussigen Phase 2. A Z Naturforsc, 1960, 15A: 287-292

[29] Onsager L. The effects of shapes on the interaction of colloidal particles. Annals of the New York Academy of Sciences, 1949, 51: 627-659

[30] Pleiner H. Alfred Saupe - 50 years of research. Liquid Crystals, 2010, 37: 619-624

[31] Rey A D, Denn M M. Dynamical phenomena in liquid-crystalline materials. Annual Review of Fluid Mechanics, 2002, 34: 233-266

[32] Wang H, Zhou H. Phase diagram of nematic polymer monolayers with the Onsager interaction potential. J Comput Theor Nanosci, 2010, 7: 738-755

[33] Wang Q, Sircar S, Zhou H. Steady state solutions of the Smoluchowski equation for rigid nematic polymers under imposed fields. Comm Math Sci, 2005, 3: 605-620

[34] Zarnescu A. The stationary 2D Smoluchowski equation in strong homogeneous flow. Nonlinearity, 2006, 19: $1619-1628$

[35] Zhou, H, Wang H, Forest M G, Wang Q. A new proof on axisymmetric equilibria of a three-dimensional Smoluchowski equation. Nonlinearity, 2005, 18: 2815-2825

[36] Zhou H, Wang H, Wang Q, Forest M G. Characterization of stable kinetic equilibria of rigid, dipolar rod ensembles for coupled dipole-dipole and Maier-Saupe potentials. Nonlinearity, 2007, 20: 277-297

[37] Zhou H, Wang H. Steady states and dynamics of 2-D nematic polymers driven by an imposed weak shear. Comm Math Sci, 2007, 5: 113-132

[38] Zhou H, Wang H, Wang Q. Nonparallel solutions of extended nematic polymers under an external field. Discrete and Continuous Dynamical Systems-Series B, 2007, 7(4): 907-929

[39] Zhou H, Forest M G, Wang H. Mathematical studies and simulations of nematic liquid crystal polymers and nanocomposites. J Comput Theor Nanosci, 2010, 7: 645-660 